\title{
Recursive POD Expansion for the Advection-Diffusion-Reaction Equation
}

\author{
M. Azaïez ${ }^{1, *}$, T. Chacón Rebollo ${ }^{2}$,E. Perracchione ${ }^{3}$ and J. M. Vega ${ }^{4}$ \\ ${ }^{1}$ Bordeaux INP, I2M (UMR CNRS 5295), Université de Bordeaux, 33607 Pessac, \\ France. \\ ${ }^{2}$ Departamento EDAN E IMUS, Universidad de Sevilla, C/Tarfia, s/n, 41012 Sevilla, \\ Spain. \\ 3 Dipartimento di Matematica Tullio Levi-Civita, Università di Padova, Padova, \\ Italy. \\ 4 E.T.S.I. Aeronáutica y del Espacio, Universidad Politécnica de Madrid, Madrid, \\ Spain.
}

Received 19 December 2017; Accepted (in revised version) 4 April 2018

\begin{abstract}
This paper deals with the approximation of advection-diffusion-reaction equation solution by reduced order methods. We use the Recursive POD approximation for multivariate functions introduced in [5] and applied to the low tensor representation of the solution of the reaction-diffusion partial differential equation. In this contribution we extend the Recursive POD approximation for multivariate functions with an arbitrary number of parameters, for which we prove general error estimates. The method is used to approximate the solutions of the advection-diffusion-reaction equation. We prove spectral error estimates, in which the spectral convergence rate depends only on the diffusion interval, while the error estimates are affected by a factor that grows exponentially with the advection velocity, and are independent of the reaction rate if this lives in a bounded set. These error estimates are based upon the analyticity of the solution of these equations as a function of the parameters (advection velocity, diffusion, reaction rate). We present several numerical tests, strongly consistent with the theoretical error estimates.
\end{abstract}

AMS subject classifications: 65D15, 65M70, 74F10

Key words: Reduced order methods, recursive POD, multivariate functions.

\section{Introduction}

Physical and real phenomena are often described by large scale problems for which a low dimensional approximation turns out to be essential. Therefore, in the last decades,

*Corresponding author. Email addresses: mejdi.azaiez@enscbp.fr (M. Azaïez), chacon@us.es (T. Chacón), emma.perracchione@math. unipd.it (E. Perracchione), josemanuel.vega@upm.es (J. M. Vega) 
ROMs, known as Karhunen-Loève expansion (KLE), [21], have gained popularity. Such techniques are also known as Proper Orthogonal Decomposition (POD), [8], in mechanical computation, Principal Component Analysis (PCA) in statistics, $[7,18]$, and Singular Value Decomposition (SVD) in linear algebra, [16]. The POD is a very general information compression technique which finds its natural applications in a wide variety of fields, such as in digital image compression, bioinformatics, signal processing and resolution of PDEs, $[3,4,6,8,19,25]$ and also the recent work of Cohen et al related to the numerical analysis of the convergence of polynomial approximation of parametric elliptic PDE's [11,12]. Hence, the problem of the tensor representation of multivariate phenomena, by means of the KLE, is a challenging computational issue.

Recently, techniques as the Proper Generalized Decomposition (PGD), [17], which gives a suitable approximation of multivariate functions by low dimensional varieties, or as the Higher Order SVD (HOSVD), $[13,14]$, have been developed. The latter provides a multilinear generalization of the best rank- $l$ approximation problem for matrices obtained by truncating the SVD. In fact, the HOSVD enables us to perform a low dimensional approximation of tensors as the SVD allows to approximate bivariate data. Also, a recent variant of the HOSVD is the Truncated Sequential HOSVD (ST-HOSVD), introduced in [26], that consists in sequentially performing a SVD analysis of the successive modes. The fact that each step for the construction of the ST-HOSVD separates two variables by the SVD procedure allows to exactly compute the truncation error in terms of the remaining singular values.

This paper focuses on the study of the low tensor representation of the solution of the reaction-diffusion partial differential equation. The Recursive POD (RPOD) was proposed in [5], as a procedure to build low dimensional representation of trivariate functions. It was applied to the diffusion-reaction equation, for which an exponential rate of convergence, depending only on the diffusion rate, was proved. In the present paper we study the extension of the RPOD to approximate the solution of the advectiondiffusion-reaction equations. To do this, we start first by proving a more general result of convergence of the RPOD expansion to approximate parametric analytic function in $L^{2}$ space. Then the analysis of the RPOD expansion of solution of reaction-diffusion partial differential equation turns to be a particular case where the parameters are advection velocity-assumed constant-, diffusion, reaction rate and space-time coordinates. We prove a spectral rate of convergence, that depends only on the diffusion rate much as in [5] for the convection-reaction equation. However here this rate is affected by a factor that exponentially grows with the Péclet number, and is independent on the reaction rate if this lives in a bounded set.

We also present some additional numerical tests that show the suitability of the RPOD to approximate analytic functions depending on a moderate number of parameters, with an exponential convergence rate.

The outline of the paper is as follows. Section 2 focuses on the presentation of the RPOD expansion to multivariate functions with an arbitrary number of parameters. In section 3 we study the application of the RPOD expansion to approximate parametric 
analytic functions valued in a $L^{2}$ space. We study the application of the RPOD to the approximation of the solution to the advection-reaction-diffusion equation in section 4 . The paper ends with section 6 where we test the convergence rates for the solution of the advection-diffusion-reaction equations.

Notation. Let $X \subset \mathbb{R}^{d}$ be a given Lipschitz domain. We denote by $L^{2}(X)$ the space of measurable and square integrable functions on $X$. The scale of fractional Sobolev spaces $H^{\tau}(X), \tau>0$ is defined as in [1]. Finally, given the sets $X_{j} \subset \mathbb{R}^{d_{j}}, j=1, \cdots, n$ we denote $\mathbf{X}_{i}^{n}=X_{i} \times X_{i+1} \times \cdots \times X_{n}$ and $\mathbf{x}_{i}^{n}=\left(x_{i}, x_{i+1}, \cdots, x_{n}\right) \in \mathbf{X}_{i}, i=1, \cdots, n$.

\section{Low dimensional tensor representation via RPOD}

This section focuses on the presentation of the RPOD expansion to multivariate functions with an arbitrary number of parameters.

\subsection{RPOD representation of multivariate functions}

In order to illustrate the RPOD representation of $n$-variate functions, at first we briefly review the basic concepts for bivariate functions, investigated in [5]. Let $X_{1} \subset \mathbb{R}^{d_{1}}$ and $X_{2} \subset$ $\mathbb{R}^{d_{2}}$ two bounded domains. The goal consists in approximating, in a low-dimensional variety, a function $f$ in the Lebesgue space $L^{2}\left(X_{1} \times X_{2}\right)$.

Given a function $f \in L^{2}\left(X_{1} \times X_{2}\right) \equiv L^{2}\left(X_{1}, L^{2}\left(X_{2}\right)\right)$, we define the integral operator $Y: L^{2}\left(X_{1}\right) \rightarrow L^{2}\left(X_{2}\right)$, with kernel $f$, as:

$$
(Y \varphi)\left(x_{2}\right)=\int_{X_{1}} f\left(x_{1}, x_{2}\right) \varphi\left(x_{1}\right) d x_{1}
$$

where $\varphi \in L^{2}\left(X_{1}\right)$. The bounded operator $Y$ has an adjoint $Y^{*}: L^{2}\left(X_{2}\right) \rightarrow L^{2}\left(X_{1}\right)$, defined as:

$$
\left(Y^{*} v\right)\left(x_{1}\right)=\int_{X_{2}} f\left(x_{1}, x_{2}\right) v\left(x_{2}\right) d x_{2}
$$

with $v \in L^{2}\left(X_{2}\right)$. Once we define the integral operator $Y$ and its adjoint, we consider the POD integral operator defined as $R=Y^{*} Y$. It follows that $R$ is a linear, bounded, nonnegative, self-adjoint and compact operator. The latter property is a consequence of the Kolmogorov compactness criterion in $L^{2}\left(X_{1}\right)$ which states that $Y^{*}$ is compact. Then, since $Y$ is bounded, it implies that $R$ is compact as well [23]. Taking into account the properties of the operator $R$, the following spectral decomposition for the POD operator holds.

Property 2.1. There exists a complete orthonormal basis of $L^{2}\left(X_{1}\right)$ consisting of eigenvectors $\left(\varphi_{i}\right)_{i \in \mathbb{N}}$ and associated non-negative real eigenvalues $\left(\lambda_{i}\right)_{i \in \mathbb{N}}$ of $R$ that we assume ordered in decreasing value. Each nonzero eigenvalue of $R$ has finite multiplicity and 0 is the only possible accumulation point of the spectrum of $R$. Moreover, $\lim _{i \rightarrow \infty} \lambda_{i}=0$. 
Concerning the computation of such basis, the sequence $\left(v_{i}\right)_{i \in \mathbb{N}}$, given by:

$$
v_{i}=\frac{1}{\sigma_{i}} Y \varphi_{i}
$$

where $\sigma_{i}=\sqrt{\lambda_{i}}$ are the so-called singular values and $\varphi_{i}=\frac{1}{\sigma_{i}} Y^{*} v_{i}$, is an orthogonal basis of $L^{2}\left(X_{2}\right)$. Since $R$ is a positive operator, by virtue of the Mercer's theorem [22], the following decomposition holds:

\section{Corollary 2.1.}

$$
f\left(x_{1}, x_{2}\right)=\sum_{i \in \mathbb{N}} \sigma_{i} \varphi_{i}\left(x_{1}\right) v_{i}\left(x_{2}\right) .
$$

The expansion (2.2) is convergent in $L^{2}\left(X_{1} \times X_{2}\right)$.

As already mentioned, the POD decomposition achieves the best approximation property, stated below.

Property 2.2. Let $V_{l}=\operatorname{span}\left(v_{1}, \cdots, v_{l}\right) \subset L^{2}\left(X_{2}\right)$ and let $W_{l}$ be any subspace of $L^{2}\left(X_{2}\right)$ of dimension $l$. Then:

$$
\int_{X_{1}} d_{L^{2}\left(X_{2}\right)}\left(f\left(x_{1}, \cdot\right), V_{l}\right)^{2} d x_{1} \leq \int_{X_{1}} d_{L^{2}\left(X_{2}\right)}\left(f\left(x_{1}, \cdot\right), W_{l}\right)^{2} d x_{1}
$$

where

$$
d_{L^{2}\left(X_{2}\right)}\left(\varphi, W_{l}\right)=\inf _{\psi \in W_{l}}\|\varphi-\psi\|_{L^{2}\left(X_{2}\right)}, \quad \varphi \in L^{2}\left(X_{2}\right)
$$

denotes the distance from the element $\varphi \in L^{2}\left(X_{2}\right)$ to the subspace $W_{l}$.

In order to formulate a recursive POD representation for multivariate functions, let $f$ be in the Lebesgue space $L^{2}\left(X_{1} \times X_{2} \times \cdots \times X_{n}\right)$, where $X_{1} \subset \mathbb{R}^{d_{1}}, X_{2} \subset \mathbb{R}^{d_{2}}, \cdots, X_{n} \subset \mathbb{R}^{d_{n}}$ are bounded domains. From Corollary 2.1 and taking into account that $L^{2}\left(\mathbf{X}_{1}^{n}\right)$ and $L^{2}\left(\mathbf{X}_{2}^{n}, L^{2}\left(X_{1}\right)\right)$ are isometric, we know that there exist two orthonormal sets $\left(\varphi_{i_{1}}\right)_{i_{1} \in \mathbb{N}}$ and $\left(v_{i_{1}}\right)_{i_{1} \in \mathbb{N}}$, which are respectively complete in $L^{2}\left(X_{1}\right)$ and $L^{2}\left(\mathbf{X}_{2}^{n}\right)$, such that:

$$
f\left(x_{1}, x_{2}, \cdots, x_{n}\right)=\sum_{i_{1} \in \mathbb{N}} \sigma_{i_{1}} v_{i_{1}}\left(x_{2}, \cdots, x_{n}\right) \varphi_{i_{1}}\left(x_{1}\right),
$$

where the sum is convergent in $L^{2}\left(\mathbf{X}_{2}^{n}, L^{2}\left(X_{1}\right)\right)$. The singular values are convergent to zero and non-negative. Now, we continue recursively applying the POD expansion to each mode $v_{i_{1}}\left(x_{2}, \cdots, x_{n}\right)$. Thus, since $L^{2}\left(\mathbf{X}_{2}^{n}\right)$ and $L^{2}\left(\mathbf{X}_{3}^{n}, L^{2}\left(X_{2}\right)\right)$ are isometric, there exist two orthonormal sets $\left(\varphi_{i_{2}}^{\left(i_{1}\right)}\right)_{i_{2} \in \mathbb{N}}$ and $\left(v_{i_{2}}^{\left(i_{1}\right)}\right)_{i_{2} \in \mathbb{N}}$, which are respectively complete in $L^{2}\left(X_{2}\right)$ and $L^{2}\left(\mathbf{X}_{3}^{n}\right)$, such that:

$$
v_{i_{1}}\left(x_{2}, \cdots, x_{n}\right)=\sum_{i_{2} \in \mathbb{N}} \sigma_{i_{2}}^{\left(i_{1}\right)} v_{i_{2}}^{\left(i_{1}\right)}\left(x_{3}, \cdots, x_{n}\right) \varphi_{i_{2}}^{\left(i_{1}\right)}\left(x_{2}\right),
$$


converges in $L^{2}\left(\mathbf{X}_{3}^{n}, L^{2}\left(X_{2}\right)\right)$. Continuing in this fashion way we find the POD expansion of $v_{i_{2}}^{\left(i_{1}\right)}, \cdots, v_{i_{n-2}}^{\left(i_{1}, i_{2}, \cdots, i_{n-3}\right)}$, where the latter is found by considering two orthonormal sets $\left(\varphi_{i_{n-1}}^{\left(i_{1}, i_{2}, \cdots, i_{n-2}\right)}\right)_{i_{n-1} \in \mathbb{N}}$ and $\left(v_{i_{n-1}}^{\left(i_{1}, i_{2}, \cdots, i_{n-2}\right)}\right)_{i_{n-1} \in \mathbb{N}}$, respectively complete in $L^{2}\left(X_{n-1}\right)$ and $L^{2}\left(X_{n}\right)$ and such that:

$$
v_{i_{n-2}}^{\left(i_{1}, i_{2}, \cdots, i_{n-3}\right)}\left(x_{n-1}, x_{n}\right)=\sum_{i_{n-1} \in \mathbb{N}} \sigma_{i_{n-1}}^{\left(i_{1}, i_{2}, \cdots, i_{n-2}\right)} v_{i_{n-1}}^{\left(i_{1}, i_{2}, \cdots, i_{n-2}\right)}\left(x_{n}\right) \varphi_{i_{n-1}}^{\left(i_{1}, i_{2}, \cdots, i_{n-2}\right)}\left(x_{n-1}\right),
$$

where the sum is convergent in $L^{2}\left(X_{n}, L^{2}\left(X_{n-1}\right)\right)$ and the singular values $\left(\sigma_{i_{n-1}}^{\left(i_{1}, i_{2}, \cdots, i_{n-2}\right)}\right)_{i_{n-1} \in \mathbb{N}}$ converge to zero and are non-negative.

Lemma 2.1. The function $f \in L^{2}\left(\mathbf{X}_{1}^{n}\right)$ admits the expansion:

$$
\begin{aligned}
f= & \sum_{i_{1} \in \mathbb{N} i_{2} \in \mathbb{N}} \sum_{i_{n-1} \in \mathbb{N}} \sigma_{i_{1}} \sigma_{i_{2}}^{\left(i_{1}\right)} \cdots \sigma_{i_{n-1}}^{\left(i_{1}, i_{2}, \cdots, i_{n-2}\right)} \varphi_{i_{1}} \otimes \varphi_{i_{2}}^{\left(i_{1}\right)} \otimes \cdots \otimes \varphi_{i_{n-1}}^{\left(i_{1}, i_{2}, \cdots, i_{n-2}\right)} \otimes v_{i_{n-1}}^{\left(i_{1}, i_{2}, \cdots, i_{n-2}\right)} \\
= & \sum_{i_{1} \in \mathbb{N}} \sigma_{i_{1}}\left(\sum_{i_{2} \in \mathbb{N}} \sigma_{i_{2}}^{\left(i_{1}\right)} \cdots\left(\sum_{i_{n-1} \in \mathcal{N}} \sigma_{i_{n-1}}^{\left(i_{1}, i_{2}, \cdots, i_{n-2}\right)} \varphi_{i_{1}} \otimes \varphi_{i_{2}}^{\left(i_{1}\right)}\right) \otimes \cdots \otimes \varphi_{i_{n-1}}^{\left(i_{1}, i_{2}, \cdots, i_{n-2}\right)}\right) \\
& \otimes v_{i_{n-1}}^{\left(i_{1}, i_{2}, \cdots, i_{n-2}\right)}
\end{aligned}
$$

where sums are convergent in $L^{2}\left(\mathbf{X}_{1}^{n}\right)$.

Proof. It is enough to prove that the series are absolutely convergent. Thus we consider the following partial absolute sum:

$$
\begin{aligned}
S_{N}= & \sum_{i_{1}=0}^{N} \sum_{i_{2}=0}^{N} \cdots \sum_{i_{n-1}=0}^{N} \| \sigma_{i_{1}} \sigma_{i_{2}}^{\left(i_{1}\right)} \cdots \sigma_{i_{n-1}}^{\left(i_{1}, i_{2}, \cdots, i_{n-2}\right)} \varphi_{i_{1}} \otimes \varphi_{i_{2}}^{\left(i_{1}\right)} \otimes \cdots \\
& \otimes \varphi_{i_{n-1}}^{\left(i_{1}, i_{2}, \cdots, i_{n-2}\right)} \otimes v_{i_{n-1}}^{\left(i_{1}, i_{2}, \cdots, i_{n-2}\right)} \|_{L^{2}\left(\mathbf{X}_{1}^{n}\right)^{n}}^{2}
\end{aligned}
$$

Taking into account that eigenfunctions are orthonormal we have:

$$
\begin{aligned}
S_{N} & =\sum_{i_{1}=0}^{N} \sum_{i_{2}=0}^{N} \cdots \sum_{i_{n-1}=0}^{N}\left|\sigma_{i_{1}}\right|^{2}\left|\sigma_{i_{2}}^{\left(i_{1}\right)}\right|^{2} \cdots\left|\sigma_{i_{n-1}}^{\left(i_{1}, i_{2}, \cdots, i_{n-2}\right)}\right|^{2} \\
& \leq \sum_{i_{1}=0}^{N} \sum_{i_{2} \in \mathbb{N}} \cdots \sum_{i_{n-1} \in \mathbb{N}}\left|\sigma_{i_{1}}\right|^{2}\left|\sigma_{i_{2}}^{\left(i_{1}\right)}\right|^{2} \cdots\left|\sigma_{i_{n-1}}^{\left(i_{1}, i_{2}, \cdots, i_{n-2}\right)}\right|^{2} \leq \sum_{i_{1}=0}^{N}\left|\sigma_{i_{1}}\right|^{2} \leq \|\left. f\right|_{L^{2}\left(\mathbf{X}_{1}^{n}\right)^{\prime}} ^{2}
\end{aligned}
$$

where the last inequality holds, since:

$$
\sum_{i_{2} \in \mathbb{N}}\left|\sigma_{i_{2}}^{\left(i_{1}\right)}\right|^{2}=\cdots=\sum_{i_{n-1} \in \mathbb{N}}\left|\sigma_{i_{n-1}}^{\left(i_{1}, i_{2}, \cdots, i_{n-2}\right)}\right|^{2}=1
$$

Then indeed the series are absolutely convergent. 
A similar result to the one outlined in Property 2.2 holds also for the RPOD:

Property 2.3. Let $f \in L^{2}\left(\mathbf{X}_{1}^{n}\right)$, then:

$$
\left\|f-f_{I}\right\|_{L^{2}\left(\mathbf{X}_{1}^{n}\right)}^{2} \leq\left\|f-\tilde{f}_{I}\right\|_{L^{2}\left(\mathbf{X}_{1}^{n}\right)^{\prime}}^{2}
$$

where

$$
f_{I}=\sum_{i=0}^{I} \sigma_{i} v_{i}\left(\mathbf{x}_{2}^{n}\right) \varphi_{i}\left(x_{1}\right)
$$

and $\tilde{f}_{I}$ is any $n$-variate approximation of $f$ with $I$ modes of the form:

$$
\tilde{f}_{I}=\sum_{i=0}^{I} \tilde{X}_{i}^{(1)}\left(x_{1}\right) \tilde{X}_{i}^{(2)}\left(x_{2}\right) \cdots \tilde{X}_{i}^{(n)}\left(x_{n}\right) .
$$

Proof. We start pointing out that $f_{I}$ is the orthogonal projection in $L^{2}\left(\mathbf{X}_{2}^{n}\right)$ of $f$ on $V_{I}$, where $V_{I}=\operatorname{span}\left(\varphi_{1}, \cdots, \varphi_{I}\right)$. Let $W_{I}$ be any other subspace of dimension $I$ of $L^{2}\left(\mathbf{X}_{2}^{n}\right)$, then from Property 2.2 it follows:

$$
\int_{X_{1}}\left\|\left(f-f_{I}\right)\left(x_{1}\right)\right\|_{L^{2}\left(\mathbf{X}_{2}^{n}\right)}^{2} d x_{1} \leq \int_{X_{1}}\left\|\left(f-\hat{f}_{I}\right)\left(x_{1}\right)\right\|_{L^{2}\left(\mathbf{X}_{2}^{n}\right)}^{2} d x_{1},
$$

for any $\hat{f}_{I}$ in $W_{I}$. Moreover since $L^{2}\left(\mathbf{X}_{1}^{n}\right)$ and $L^{2}\left(X_{1}, L^{2}\left(\mathbf{X}_{2}^{n}\right)\right)$ are isometric, (2.6) follows taking $\hat{f}_{I}=\tilde{f}_{I}$.

Remark 2.1. The algebraic version of the RPOD is a particular case of the so called hierarchical tensor representation (see [28]). The link between the two approaches can be established in the same way as for the POD and SVD. See Volkwein [27] for the mathematical analysis.

\section{RPOD applied to parametric analytic functions}

In this section we study the application of the RPOD expansion to approximate parametric analytic functions valued in a $L^{2}$ space. We intend to apply the results of this section to the approximation of the solution of the advection-diffusion-reaction equation, among other equations.

Let us consider an open set $\mathcal{Q} \subset \mathbb{R}^{m_{1}}$, a tensor parameter set $\mathcal{G}=\prod_{i=2}^{n} G_{i} \subset \mathbb{R}^{m_{2}} \times \cdots \times \mathbb{R}^{m_{n-1}} \times \mathbb{C}$ and an analytic function $f: \mathcal{G} \mapsto L^{2}(\mathcal{Q})$. We shall denote by $x_{1}$ the elements of $\mathcal{Q}$ and by $x_{i}$ the elements of $G_{i}, i=2,3, \cdots, n$. We further assume that all the $G_{i}$ are compact, and that $G_{n}$ contains some ellipse

$$
\mathcal{E}_{\rho}=\left\{\zeta \in \mathbb{C}:|| \zeta-1|+| \zeta+1 \mid \leq \rho+\rho^{-1}\right\}
$$


contained in the half right complex plane, that is $\mathcal{E}_{\rho} \subset \mathbb{C}^{+}=\{\zeta \in \mathbb{C} \mid \operatorname{Re} \zeta>0\}$.

Our purpose is to build an approximation of $f$, named $f_{P}$, by truncating the POD expansion, defined as:

$$
f_{P}=\sum_{i_{1}=0}^{M_{1}} \sigma_{i_{1}} \sum_{i_{2}=0}^{M_{2}} \sigma_{i_{2}}^{\left(i_{1}\right)} \cdots \sum_{i_{n-1}=0}^{M_{n-1}} \sigma_{i_{n-1}}^{\left(i_{1}, i_{2}, \cdots, i_{n-2}\right)} \varphi_{i_{1}} \otimes \varphi_{i_{2}}^{\left(i_{1}\right)} \otimes \cdots \otimes \varphi_{i_{n-1}}^{\left(i_{1}, i_{2}, \cdots, i_{n-2}\right)} \otimes v_{i_{n-1}}^{\left(i_{1}, i_{2}, \cdots, i_{n-2}\right)} .
$$

The notation $P$ is a multi-index notation and stands for $P=\left(M_{1}, M_{2}, \cdots, M_{n-1}\right)$.

The estimation of rate of convergence of the truncated expansion $f_{P}$ towards $f$ is stated as follows

Theorem 3.1. The truncated RPOD series expansion $f_{P}$ given by (3.1) satisfies the error estimate

$$
\left\|f-f_{P}\right\|_{L^{2}(\mathcal{G} \times \mathcal{Q})} \leq C_{\rho} K\left(\rho^{-M_{1}}+\sqrt{M_{1}} \rho^{-M_{2}}+\cdots+\sqrt{M_{1} M_{2} \cdots M_{n-1}} \rho^{-M_{n-1}}\right),
$$

for any $1<\rho<\rho_{*}$, where $C_{\rho}=\frac{2}{\rho-1}|\mathcal{G}|^{1 / 2}$, and $K=\max _{\left(x_{2}, \cdots, x_{n}\right) \in \mathcal{G}}\left\|f\left(x_{2}, \cdots, x_{n}\right)\right\|_{L^{2}(\mathcal{Q})}$.

Therefore, the RPOD expansion converges with spectral accuracy in terms of the number of truncation modes in the main, secondary and tertiary expansions.

Our analysis is based upon the estimates for rate of convergence of polynomial interpolation of vector-valued functions. We shall adapt a result by S. Bernstein in 1912, improved since then in many works (see for instance [20]). For some $\rho>1$, let the ellipse $E_{\rho}$ in the complex plane be defined as

$$
E_{\rho}=\left\{\zeta \in \mathbf{C}:|| \zeta-1|+| \zeta+1 \mid \leq \rho+\rho^{-1}\right\} .
$$

Consider a function $F: E_{\rho} \rightarrow H$ where $H$ is a Hilbert space. For a given integer number $M \geq 0$ let $F_{M}$ be the truncated Chebyshev polynomial series expansion of $F$ of degree $M$ with coefficients in $H$. Then we have (cf. [24, Theorem 8.2]),

Lemma 3.1. Assume that $F$ is analytic and bounded in $E_{\rho}$. There holds that

$$
\max _{\xi \in[-1,1]}\left\|F(\xi)-F_{M}(\xi)\right\|_{H} \leq C_{\rho} \rho^{-M},
$$

where $C_{\rho}=\frac{2}{\rho-1}\|F\|_{L^{\infty}\left(E_{\rho}, H\right)}$.

Proof of Theorem 3.1. Let us consider the truncated primary expansion

$$
f_{M_{1}}\left(x_{1}, x_{2}, x_{3}, \cdots, x_{n}\right)=\sum_{i_{1}=0}^{M_{1}} \sigma_{i_{1}} v_{i_{1}}\left(x_{2}, x_{3}, \cdots, x_{n}\right) \varphi_{i_{1}}\left(x_{1}\right),
$$

for some integer $M_{1} \geq 0$. We adapt the proof of Lemma 0.10 in [5]. By hypothesis, for any given $\left(x_{2}, x_{3}, \cdots, x_{n-1}\right) \in G_{2} \times G_{3} \times \cdots \times G_{n-1}$, the vector-valued function

$$
\zeta \in G_{4} \mapsto f\left(\cdot, x_{2}, x_{3}, \cdots, x_{n-1}, \zeta\right) \in L^{2}(\mathcal{Q})
$$


is analytic in the ellipse $\mathcal{E}_{\rho}$. For brevity of notation we denote this function by $f\left(x_{2}, x_{3}, \cdots, x_{n-1}, \zeta\right)$. Consider the change of coordinates

$$
\zeta=\tau(\hat{\zeta}):=\frac{\bar{\gamma}-\underline{\gamma}}{2} \hat{\zeta}+\frac{\bar{\gamma}+\underline{\gamma}}{2}, \quad \hat{\zeta} \in E_{\rho} .
$$

It is affine and bijective from $E_{\rho}$ into $\mathcal{E}_{\rho}$ and transforms the reference interval $[-1,1]$ into $[\underline{\gamma}, \bar{\gamma}]$. Let $\hat{S}_{M_{1}}^{\left(x_{2}, x_{3}, \cdots, x_{n-1}\right)}(\hat{\zeta})$ be the truncated Chebyshev series expansion of the (transformed) function $\hat{f}^{\left(x_{2}, x_{3}, \cdots, x_{n-1}\right)}(\hat{\zeta})=f\left(x_{2}, x_{3}, \cdots, x_{n-1}, \zeta\right)$. Let $S_{M_{1}}^{\left(x_{2}, x_{3}, \cdots, x_{n-1}\right)}(\zeta)=$ $\hat{S}_{M_{1}}^{\left(x_{2}, x_{3}, \cdots, x_{n-1}\right)}(\hat{\zeta})$.

From Lemma 3.1 we obtain

$$
\begin{aligned}
& \max _{\gamma \in[\underline{\gamma}, \bar{\gamma}]}\left\|f\left(x_{2}, x_{3}, \cdots, x_{n}\right)-S_{M_{1}}^{\left(x_{2}, x_{3}, \cdots, x_{n-1}\right)}\left(x_{4}\right)\right\|_{L^{2}(\mathcal{Q})} \\
\leq & \frac{2}{\rho-1}\left\|f\left(x_{2}, x_{3}, \cdots, x_{n-1}, \cdot\right)\right\|_{L^{\infty}\left(\mathcal{E}_{\rho}, L^{2}(\mathcal{Q})\right)} \rho^{-M_{1}} \leq \frac{2 K}{\rho-1} \rho^{-M_{1}} .
\end{aligned}
$$

In view of the best approximation property stated in Lemma 2.2, it holds

$$
\begin{aligned}
\left\|f-f_{M_{1}}\right\|_{L^{2}(\mathcal{G} \times \mathcal{Q})} & \leq\left\|T-S_{M_{1}}\right\|_{L^{2}(\mathcal{G} \times \mathcal{Q})} \\
& \leq|\mathcal{G}|^{1 / 2} \max _{\left(x_{2}, x_{3}, \cdots, x_{n}\right) \in \mathcal{G}}\left\|f\left(x_{2}, x_{3}, \cdots, x_{n}\right)-S_{M_{1}}^{\left(x_{2}, x_{3}, \cdots, x_{n-1}\right)}\left(x_{n}\right)\right\|_{L^{2}(\mathcal{Q})} .
\end{aligned}
$$

By estimate (3.3) it follows that

$$
\left\|f-f_{M_{1}}\right\|_{L^{2}(\mathcal{G} \times \mathcal{Q})} \leq C_{\rho} K \rho^{-M_{1}}, \quad \text { where } \quad C_{\rho}=\frac{2|\mathcal{G}|^{1 / 2}}{\rho-1} .
$$

Let us now consider the secondary expansion

$$
f_{\left(M_{1}, M_{2}\right)}\left(x_{1}, x_{2}, x_{3}, \cdots, x_{n}\right)=\sum_{i_{1}=0}^{M_{1}} \sum_{i_{2}=0}^{M_{2}} \sigma_{i_{1}} \sigma_{i_{2}}^{\left(i_{1}\right)} \varphi_{i_{1}}\left(x_{1}\right) \varphi_{i_{2}}^{\left(i_{1}\right)}\left(x_{2}\right) v_{i_{2}}^{\left(i_{1}\right)}\left(x_{3}, \cdots, x_{n}\right) .
$$

As the sequence $\left(\varphi_{i_{1}}\right)_{m \geq 0}$ is orthonormal in $L^{2}(\mathcal{Q})$, then

$$
\left\|f_{M_{1}}-f_{\left(M_{1}, M_{2}\right)}\right\|_{L^{2}(\mathcal{G} \times \mathcal{Q})}^{2} \leq \sum_{i_{1}=0}^{M_{1}} \sigma_{i_{1}}^{2}\left\|v_{i_{1}}-v_{i_{1}}^{\left(M_{2}\right)}\right\|_{L^{2}(\mathcal{G})^{\prime}}^{2}
$$

where

$$
v_{i_{1}}^{\left(M_{2}\right)}\left(x_{2}, x_{3}, \cdots, x_{n}\right)=\sum_{i_{2}=0}^{M_{2}} \sigma_{i_{2}}^{\left(i_{1}\right)} \varphi_{i_{2}}^{\left(i_{1}\right)}\left(x_{2}\right) v_{i_{2}}^{\left(i_{1}\right)}\left(x_{3}, \cdots, x_{n}\right)
$$

is the truncated POD expansion of $v_{i_{1}}$ to $M_{2}+1$ terms. 
As

$$
v_{i_{1}}\left(x_{2}, x_{3}, \cdots, x_{n}\right)=\frac{1}{\sigma_{i_{1}}} \int_{\mathcal{Q}} f\left(x_{1}, x_{2}, x_{3}, \cdots, x_{n}\right) \varphi_{i_{1}}\left(x_{1}\right) d x_{1} d t
$$

then $v_{i_{1}}$ is an analytic function from $\mathcal{G}$ into $\mathbb{R}$. By an argument similar to the one that has lead to estimate (3.3), we prove that for any $\left(x_{2}, x_{3}, \cdots, x_{n-1}\right) \in G_{2} \times G_{3} \times \cdot \times G_{n-1}$ there exists a polynomial in $x_{n}, S_{M_{2}}^{\left(i_{1}, x_{2}, x_{3}, \cdots, x_{n-1}\right)}\left(x_{n}\right)$, of degree less or equal than $M_{2}$ such that

$$
\begin{aligned}
& \max _{x_{n} \in G_{n}}\left|v_{i_{1}}\left(x_{2}, x_{3}, \cdots, x_{n}\right)-S_{M_{2}}^{\left(i_{1}, x_{2}, x_{3}, \cdots, x_{n-1}\right)}\left(x_{n}\right)\right| \\
\leq & \frac{2}{1-\rho}\left\|v_{i_{1}}\left(x_{2}, x_{3}, \cdots, x_{n-1}, \cdot\right)\right\|_{L^{\infty}\left(\mathcal{E}_{\mathcal{C}}\right)} \rho^{-M_{2}} .
\end{aligned}
$$

From (3.6), we deduce $\left|\sigma_{i_{1}}\right|\left|v_{i_{1}}\left(x_{2}, x_{3}, \cdots, x_{n}\right)\right| \leq\left\|f\left(\cdot, x_{2}, x_{3}, \cdots, x_{n}\right)\right\|_{L^{2}(\mathcal{Q})}$ for all $\left(x_{2}, x_{3}, \cdots, x_{n}\right) \in \mathcal{G}$, and then

$$
\left|\sigma_{i_{1}}\right| \max _{\left(x_{2}, x_{3}, \cdots, x_{n}\right) \in \mathcal{G}}\left|v_{i_{1}}\left(x_{2}, x_{3}, \cdots, x_{n}\right)-S_{M_{2}}^{\left(i_{1}, x_{2}, x_{3}, \cdots, x_{n-1}\right)}\left(x_{n}\right)\right| \leq \frac{2}{1-\rho} K \rho^{-M_{2}},
$$

Consequently, by the best approximation property,

$$
\begin{aligned}
\left|\sigma_{i_{1}}\right|\left\|v_{i_{1}}-v_{i_{1}}^{\left(M_{2}\right)}\right\|_{L^{2}(\mathcal{G})} & \leq\left|\sigma_{i_{1}}\right|\left\|v_{i_{1}}-S_{M_{2}}^{\left(i_{1}\right)}\right\|_{L^{2}(\mathcal{G})} \\
& \leq|\mathcal{G}|^{1 / 2}\left|\sigma_{i_{1}}\right|\left\|v_{i_{1}}-S_{M_{2}}^{\left(i_{1}\right)}\right\|_{L^{\infty}(\mathcal{G})} \leq C_{\rho} K \rho^{-M_{2}}
\end{aligned}
$$

where $S_{M_{2}}^{\left(i_{1}\right)}\left(x_{2}, x_{3}, \cdots, x_{n}\right)=S_{M_{2}}^{\left(i_{1}, x_{2}, x_{3}, \cdots, x_{n-1}\right)}\left(x_{n}\right)$. Therefore

$$
\left\|f_{M_{1}}-f_{\left(M_{1}, M_{2}\right)}\right\|_{L^{2}(\mathcal{G} \times \mathcal{Q})} \leq C_{\rho} \sqrt{M_{1}} K \rho^{-M_{2}} .
$$

Let us consider next the tertiary expansion,

$$
f_{\left(M_{1}, M_{2}, M_{3}\right)}=\sum_{i_{1}=0}^{M_{1}} \sum_{i_{2}=0}^{M_{2}} \sum_{i_{3}=0}^{M_{3}} \sigma_{i_{1}} \sigma_{i_{2}}^{\left(i_{1}\right)} \sigma_{i_{3}}^{\left(i_{1}, i_{2}\right)} \varphi_{i_{1}}\left(x_{1}\right) \varphi_{i_{2}}^{\left(i_{1}\right)}\left(x_{2}\right) \varphi_{i_{3}}^{\left(i_{1}, i_{2}\right)}\left(x_{3}\right) v_{i_{3}}^{\left(i_{1}, i_{2}\right)}\left(x_{4}, \cdots, x_{n}\right) .
$$

As the functions $\varphi_{i_{1}}\left(x_{1}\right) \varphi_{i_{2}}^{\left(i_{1}\right)}\left(x_{2}\right)$ are orthogonal in $L^{2}\left(G_{1} \times G_{2}\right)$, it holds

$$
\left\|f_{\left(M_{1}, M_{2}\right)}-f_{\left(M_{1}, M_{2}, M_{3}\right)}\right\|_{L^{2}(\mathcal{G} \times \mathcal{Q})}^{2} \leq \sum_{i_{1}=0}^{M_{1}} \sum_{i_{2}=0}^{M_{2}} \sigma_{i_{1}}^{2}\left|\sigma_{i_{2}}^{\left(i_{1}\right)}\right|^{2}\left\|v_{i_{2}}^{i_{1}}-v_{i_{2}}^{\left(i_{1}, M_{3}\right)}\right\|_{L^{2}\left(G_{3} \times \cdots \times G_{n}\right)^{\prime}}^{2}
$$

where

$$
v_{i_{2}}^{\left(i_{1}, M_{3}\right)}\left(x_{3}, \cdots, x_{n}\right)=\sum_{i_{3}=0}^{M_{3}} \sigma_{i_{3}}^{\left(i_{1}, i_{2}\right)} \varphi_{i_{3}}^{\left(i_{1}, i_{2}\right)}\left(x_{3}\right) v_{i_{3}}^{\left(i_{1}, i_{2}\right)}\left(x_{4}, \cdots, x_{n}\right)
$$


is the truncated expansion of $v_{i_{2}}^{i_{1}}$ to $M_{3}+1$ terms. As

$$
\begin{aligned}
v_{i_{2}}^{\left(i_{1}\right)}\left(x_{3}, \cdots, x_{n}\right) & =\frac{1}{\sigma_{i_{2}}^{\left(i_{1}\right)}} \int_{G_{2}} v_{i_{1}}\left(x_{2}, x_{3}, \cdots, x_{n}\right) \varphi_{i_{2}}^{\left(i_{1}\right)}\left(x_{2}\right) d x_{2} \\
& =\frac{1}{\sigma_{i_{2}}^{\left(i_{1}\right)}} \frac{1}{\sigma_{i_{1}}} \int_{G_{2}} \int_{Q} f\left(x_{1}, x_{2}, x_{3}, \cdots, x_{n}\right) \varphi_{i_{1}}\left(x_{1}\right) \varphi_{i_{2}}^{\left(i_{1}\right)}\left(x_{2}\right) d x_{1} d x_{2}
\end{aligned}
$$

then $v_{i_{2}}^{\left(i_{1}\right)}$ is an analytic function from $G_{3} \times \cdots \times G_{n}$ into $\mathbb{R}$. Then, similarly as for $v_{i_{1}}$, there exists a polynomial $S_{M_{3}}^{\left(i_{1}, i_{2}, x_{3}, \cdots, x_{n-1}\right)}\left(x_{4}\right)$ of degree $\leq M_{3}+1$ such that

$$
\max _{x_{n} \in G_{n}}\left|v_{i_{2}}^{\left(i_{1}, M_{3}\right)}\left(x_{3}, \cdots, x_{n}\right)-S_{M_{3}}^{\left(i_{1}, i_{2}, x_{3}, \cdots, x_{n-1}\right)}\left(x_{n}\right)\right| \leq \frac{2}{1-\rho}\left\|v_{i_{2}}^{\left(i_{1}\right)}\left(x_{3}, \cdots, x_{n}\right)\right\|_{L^{\infty}\left(\mathcal{E}_{C}\right)} \rho^{-M_{3}} .
$$

By (3.11), $\left|\sigma_{i_{2}}^{\left(i_{1}\right)}\right|\left|\sigma_{i_{1}}\right|\left|v_{i_{2}}^{\left(i_{1}\right)}\left(x_{3}, \cdots, x_{n}\right)\right| \leq\left\|f\left(x_{2}, x_{3}, \cdots, x_{n}\right)\right\|_{L^{2}(\mathcal{Q})} \leq K$. Then it follows

$$
\left|\sigma_{i_{2}}^{\left(i_{1}\right)}\left\|\sigma_{i_{1}} \mid\right\| v_{i_{2}}^{\left(i_{1}\right)}-v_{i_{2}}^{\left(i_{1}, M_{3}\right)} \|_{L^{2}\left(G_{3} \times \cdots \times G_{n}\right)} \leq C_{\rho} K \rho^{-M_{3},}\right.
$$

where $\tilde{C}_{\rho}$ is a constant, possibly unbounded as $\rho \rightarrow 1$. Then (3.10) implies

$$
\left\|f_{\left(M_{1}, M_{2}\right)}-f_{\left(M_{1}, M_{2}, M_{3}\right)}\right\|_{L^{2}(\mathcal{G} \times \mathcal{Q})} \leq C_{\rho} \sqrt{M_{1} M_{2}} K \rho^{-M_{3}} .
$$

Similarly, the difference between two consecutive partial expansions (with obvious notation) is bounded by

$$
\left\|f_{\left(M_{1}, M_{2}, \cdots, M_{k-1}\right)}-f_{\left(M_{1}, M_{2}, \cdots, M_{k}\right)}\right\|_{L^{2}(\mathcal{G} \times \mathcal{Q})} \leq C_{\rho} \sqrt{M_{1} M_{2} \cdots, M_{k-1}} K \rho^{-M_{k}} .
$$

Combining (3.5), (3.9), (3.12) and (3.13), estimate (4.7) follows.

\section{Applications}

In this section we study the application of the RPOD expansion to approximate the solutions of parametric ODEs and PDEs, and in particular of the parametric linear advectiondiffusion-reaction equations.

We are particularly interested in the linear advection-diffusion-reaction equation as it governs a number of phenomena of interest (heat transfer, pollutant behavior in fluids, future value of assets in Black-Scholes theory, etc.). Let us consider the homogeneous Dirichlet boundary value problem for the linear advection-diffusion-reaction equation in a bounded domain $\Omega \subset \mathbb{R}^{d}, d \geq 1$, and a time interval $\left(0, t^{*}\right), t^{*}>0$ :

$$
\begin{cases}\partial_{t} u+\vec{a} \cdot \nabla u-\gamma \Delta u+\alpha u=f, & \text { in } \mathcal{Q}, \\ u=0, & \text { in }\left(0, t^{*}\right) \times \partial \Omega, \\ u(x, 0)=u_{0}(x), & \text { in } \Omega,\end{cases}
$$


where $\vec{a} \in \mathbb{R}^{3}$ is the advection velocity field, that we assume constant, $\gamma>0$ and $\alpha \geq 0$ respectively denote the diffusivity and the reaction rate, and $\mathcal{Q}=\Omega \times\left(0, t^{*}\right)$. This problem fits into the functional framework of constant-coefficient linear parabolic equations, and admits a unique solution $u \in L^{2}\left(\left(0, t^{*}\right), H^{1}(\Omega)\right)$ such that $\partial_{t} u \in L^{2}(\mathcal{Q})$ if $f \in L^{2}(\mathcal{Q})$ and $u_{0} \in L^{2}(\Omega)$. We shall denote by $\Lambda$ the diameter of $\Omega$ and assume that the triad $(\vec{a}, \alpha, \gamma)$ ranges in a set $\mathcal{G}=B_{R} \times[0, \bar{\alpha}] \times[\underline{\gamma}, \bar{\gamma}]$ where $B_{R} \subset \mathbb{R}^{3}$ is the ball of radius $R>0$, and $0<\underline{\gamma}<\bar{\gamma}$, $0 \leq \bar{\alpha}$. It holds that

Lemma 4.1. The mapping $(\vec{a}, \alpha, \gamma) \in \mathcal{G} \mapsto u_{(\vec{a}, \alpha, \gamma)} \in L^{2}(\mathcal{Q})$ is analytic, and

$$
\max _{(\vec{a}, \alpha, \gamma) \in \mathcal{G}}\|u(\vec{a}, \alpha, \gamma)\|_{L^{2}(\mathcal{Q})} \leq C e^{R \Lambda /} \underline{\gamma}
$$

$C$ is a constant depending of the data $u_{0}$ and $f$.

Proof. Let us make the change of variable (we omit the dependence upon the parameters for brevity)

$$
u(x, t)=e^{\vec{a} \cdot x /(2 \gamma)} W(x, t) .
$$

A straightforward calculation shows that $W$ is the solution of an initial-boundary condition problem for the advection-diffusion-reaction equation,

$$
\begin{cases}\partial_{t} W-\gamma \Delta W+\sigma W=g, & \text { in } \mathcal{Q}, \\ W=0, & \text { in }\left(0, t^{*}\right) \times \partial \Omega, \\ W(x, 0)=e^{-\vec{a} \cdot x /(2 \gamma)} u_{0}(x), & \text { in } \Omega,\end{cases}
$$

where $\sigma=\alpha+\frac{1}{2}|a|^{2}$ and $g(x, t)=e^{-\vec{a} \cdot x /(2 \gamma)} f(x, t)$. Let us define the constant

$$
\rho_{*}=(r+1) /(r-1), \quad \text { with } r=\sqrt{\bar{\gamma} / \underline{\gamma}} .
$$

In [5] it is proved that the mapping $(\gamma, \sigma) \in \mathcal{E}_{\rho^{*}} \times[0, \bar{\sigma}] \mapsto W_{(\gamma, \sigma)} \in L^{2}(\mathcal{Q})$ is analytic, for any $\bar{\sigma} \geq 0$. Consequently, the mapping $(\vec{a}, \alpha, \gamma) \in \mathcal{G} \mapsto u_{(\vec{a}, \alpha, \gamma)} \in L^{2}(\mathcal{Q})$ is analytic. Let

$$
K=\max _{(\vec{a}, \alpha, \gamma) \in \mathcal{G}}\|u(\vec{a}, \alpha, \gamma)\|_{L^{2}(\mathcal{Q})} .
$$

Due to (4.3),

$$
\begin{aligned}
K & \leq \sup _{(\vec{a}, x, \gamma) \in B_{R} \times \Omega \times[\underline{\gamma}, \bar{\gamma}]} e^{\vec{a} \cdot x /(2 \gamma)} \sup _{\sigma \in\left[0, \bar{\alpha}+R^{2} / 2\right]}\|W(\cdot, \sigma)\|_{L^{\infty}\left(\mathcal{E}_{\rho}, L^{2}(\mathcal{Q})\right)} \\
& \leq c e^{R \Lambda / \underline{\gamma}}\left(\sqrt{t^{*}}\left\|u_{0}\right\|_{L^{2}(\Omega)}+t^{*}\|f\|_{L^{2}(Q)}\right),
\end{aligned}
$$

where the last inequality follows from a standard estimate for the solution of (4.4) (we omit the dependence with respect to the parameters):

$$
\|W\|_{L^{2}(Q)} \leq c\left(\sqrt{t^{*}}\|W(0)\|_{L^{2}(\Omega)}+t^{*}\|g\|_{L^{2}(Q)}\right),
$$

where $c$ is a constant that depends only on $\Omega$. Thus (4.2) follows. 
Remark 4.1. Note that the quantity $R \Lambda / \gamma$ that appears in estimate (4.2) is some kind of global Péclet number for all velocities $\vec{a} \in \bar{B}_{R}$ and diffusions $\gamma \in[\underline{\gamma}, \bar{\gamma}]$.

We may now apply Theorem 3.1 to the function $\mathfrak{u}$, taking $n=4, m_{1}=4, m_{2}=3, m_{3}=1$ and $m_{4}=1, x_{1}=(x, t), x_{2}=\vec{a}, x_{3}=\alpha$ and $x_{4}=\gamma$. Then it follows

$$
\left\|u-u_{\left(M_{1}, M_{2}, M_{3}\right)}\right\|_{L^{2}(\mathcal{G} \times \mathcal{Q})} \leq C_{\rho} e^{R \Lambda / \underline{\gamma}}\left(\rho^{-M_{1}}+\sqrt{M_{1}} \rho^{-M_{2}}+\sqrt{M_{1} M_{2}} \rho^{-M_{3}}\right),
$$

for any $1<\rho<\rho_{*}$, where $C_{\rho}>0$ is a constant depending on $\rho$, unbounded as $\rho \rightarrow 1$. Note that he rate of convergence depends on the ratio $\bar{\gamma} / \underline{\gamma}$, while there is an amplification factor that grows exponentially with the velocity.

This result in particular includes that of [4] that studies the RPOD approximation to the diffusion-reaction equation, in our present framework $\vec{a}=\overrightarrow{0}$.

In general, parametric PDEs whose solution has an analytic dependence with respect to the parameters can also be approximated with spectral accuracy by the RPOD method.

Let us now consider the Cauchy problem for parametric ODEs. Let $T>0, \mathcal{Y} \subset \mathbb{R}^{k}$ an open connected set and $\Lambda \subset \mathbb{R}^{l}$ a domain for the parameters. Consider the problem

$$
y^{\prime}=\phi(t, y, \lambda) ; \quad y\left(t_{0}\right)=y_{0},
$$

where $\phi:(0, T) \times \mathcal{Y} \times \Lambda \mapsto \mathbb{R}^{k}$ is a continuous function, uniformly (in $\left.\lambda\right)$ locally Lipschitz with respect to $y$ in a neighborhood of $\left(t_{0}, y_{0}\right)$, and with an analytic dependence with respect to $\lambda$. In this case the solution $y(t, \lambda)$ also presents an analytic dependence with respect to $\lambda$ (cf. [2]). If the domain of analyticity can be extended (with respect to some component $\lambda_{i}$ ) to a compact sub-set of $\mathbb{C}^{+}$containing an ellipse $\mathcal{E}_{\rho}$, then the function $y(t, \lambda)$ fits the theoretical framework of Theorem 3.1.

In this case the function $y(t, \lambda)$ is approximated by the RPOD with spectral accuracy. Then if the number of parameters is moderate, a reduced number of modes suffices to provide a good accuracy.

\section{Some practical issues}

In this section we analyze some questions related to the practical implementation of the RPOD. A relevant question treated is the determination of the number of modes required to meet a prescribed accuracy. Another is the understanding of the convergence pattern, that we explain by means of a re-ordering of the POD expansion in terms of decreasing size of modes. We analyze both questions for general analytic functions and apply the analysis carried on to the solution of the advection-reaction-diffusion equation.

\subsection{Convergence pattern of RPOD expansion for analytic functions}

A natural re-ordering of expansion (2.4) is in a decreasing order of the values $\tilde{\sigma}_{\alpha}=$ $\sigma_{i_{1}} \sigma_{i_{2}}^{i_{1}} \cdots \sigma_{i_{n-1}}^{\left(i_{1}, i_{2}, \cdots, i_{n-2}\right)}$, where we use the multi-index notation $\alpha=\left(i_{1}, \cdots, i_{n-1}\right) \in \mathcal{N}^{n-1}$. Ex- 
pansion (2.4) may be written as

$$
f=\sum_{k \geq 1|\alpha|=k} \sum_{\alpha} \tilde{\sigma}_{\alpha}
$$

where $|\alpha|=i_{1}+i_{2}+\cdots+i_{n-1}$ and

$$
\Psi_{\alpha}=\varphi_{i_{1}} \otimes \varphi_{i_{2}}^{\left(i_{1}\right)} \otimes \cdots \otimes \varphi_{i_{n-1}}^{\left(i_{1}, i_{2}, \cdots, i_{n-2}\right)} \otimes v_{i_{n-1}}^{\left(i_{1}, i_{2}, \cdots, i_{n-2}\right)} .
$$

In practice frequently it holds

$$
\tilde{\sigma}_{\alpha} \simeq \rho^{-|\alpha|} \text { with } \rho>1
$$

for smooth functions $f$. Then the coefficients $\sigma_{\alpha}$ in expansion (5.1) indeed decrease as $k=|\alpha|$ increases. This property in practice may be determined for a given function when calculating its RPOD expansion. This allows to find a practical error estimate for the truncated expansion

$$
f_{K}=\sum_{k=1}^{K} \sum_{|\alpha|=k} \tilde{\sigma}_{\alpha} \Psi_{\alpha}
$$

The associated error is estimated by

$$
\left\|f-f_{K}\right\|_{L^{2}\left(x_{1}^{n}\right)} \leq D_{n} K^{\frac{n-2}{2}} \rho^{-K}
$$

where $D_{n}$ is a constant. To justify it we use that the functions $\Psi_{\alpha}$ are orthonormal in $L^{2}\left(\mathbf{X}_{1}^{n}\right)$. Denoting $\mathcal{A}_{n, k}=\left\{\alpha \in \mathcal{N}^{n-1}:|\alpha|=k\right\}$ and $C$ a positive constant, this yields

$$
\left\|f-f_{K}\right\|_{L^{2}\left(\mathbf{X}_{1}^{n}\right)}^{2} \leq \sum_{k \geq K+1|\alpha|=k}\left|\tilde{\sigma}_{\alpha}\right|^{2} \leq C \sum_{k \geq K+1} \operatorname{card}\left(\mathcal{A}_{n, k}\right) \rho^{-2 k}=C \sum_{k \geq K+1}\left(\begin{array}{c}
n+k-2 \\
k
\end{array}\right) \rho^{-2 k},
$$

where we use that

$$
\operatorname{card}\left(\mathcal{A}_{n, k}\right)=\left(\begin{array}{c}
n+k-2 \\
k
\end{array}\right)
$$

Let us remember that

$$
\left(\begin{array}{l}
n \\
k
\end{array}\right)=\frac{1}{(n+1) B(n-k+1, k+1)},
$$

where $B$ is the $\beta$ function. As the Stirling formula yields

$$
B(x, y) \simeq \frac{1}{\sqrt{2 \pi}} \frac{x^{x-1 / 2} y^{y-1 / 2}}{(x+y)^{x+y-1 / 2}}
$$

it follows

$$
\left(\begin{array}{c}
n+k-2 \\
k
\end{array}\right) \simeq \frac{1}{\sqrt{2 \pi}} \frac{1}{n+k-1} \frac{(n+k)^{n+k-1 / 2}}{(n-1)^{n-3 / 2}(k+1)^{k+1 / 2}} \simeq C_{n} k^{n-2}
$$


Then,

$$
\left\|f-f_{K}\right\|_{L^{2}\left(\mathbf{x}_{1}^{n}\right)}^{2} \leq C C_{n} \sum_{k \geq K+1} k^{n-2} \rho^{-2 k} \leq C_{n}^{\prime} K^{n-2} \rho^{-2 K},
$$

where the last estimate is obtained by comparison of the sum $\sum_{k \geq K+1} k^{n-2} \rho^{-2 k}$ with $\int_{K}^{\infty} s(x) d x$, with $s(x)=x^{n-2} \rho^{-2 x}$, which is decreasing for $x>(n-2) / 2$. Then (5.6), and consequently (5.5), follow.

Let us re-order now the expansion (5.4) in decreasing order of the coefficients $\tilde{\sigma}_{\alpha}$, as

$$
f_{K}=\sum_{l=0}^{L_{K}} \hat{\sigma}_{l} \hat{\Psi}_{l},
$$

where

$$
L_{K}=\sum_{k=1}^{K} \operatorname{card}\left(\mathcal{A}_{n, k}\right)=\left(\begin{array}{c}
n+K-1 \\
K
\end{array}\right)
$$

and respectively the coefficients $\hat{\sigma}_{l}$ and the functions $\hat{\Psi}_{l}$ are the re-ordered coefficients $\sigma_{\alpha}$ in decreasing value, and the functions $\Psi_{\alpha}$ defined by (5.2). Then $L_{K} \simeq \frac{1}{(n-1) !} K^{n-1}$, and (5.5) yields an estimate of the $L^{2}$ error in terms of the number of modes in expansion (5.7),

$$
\left\|f-f_{K}\right\|_{L^{2}\left(\mathbf{X}_{1}^{n}\right)} \leq D_{n}^{\prime} L_{K}^{\frac{n-2}{2(n-1)}} \rho^{-L_{K}^{\frac{1}{n-1}}} .
$$

The analysis carried on in the proof of Theorem 3.1 may be applied to estimate the rate of convergence of the re-arranged RPOD expansion (5.1) for $u$. Indeed, denote by $\mathcal{L}(E, F)$ the set of linear bounded mappings from a Banach space $E$ into a Banach space $F$; the following bound holds,

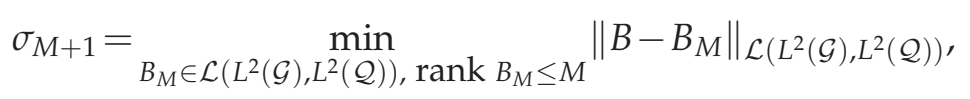

where

$$
(B \varphi)(z)=\int_{\underline{\gamma}}^{\bar{\gamma}} u(\vec{a}, \alpha, \gamma, z) \varphi(\gamma) d \gamma, \quad \forall z \in \mathcal{Q} .
$$

Consider the operator

$$
\left(\tilde{B}_{M} \varphi\right)(z)=\int_{\underline{\gamma}}^{\bar{\gamma}} u_{M}(\vec{a}, \alpha, \gamma, z) \varphi(\gamma) d \gamma, \quad \forall z \in \mathcal{Q} .
$$

Then by estimate (3.5)

$$
\sigma_{M+1} \leq\left\|B-\tilde{B}_{M}\right\|_{\mathcal{L}\left(L^{2}(\mathcal{G}), L^{2}(\mathcal{Q})\right)}=\left\|u-u_{M}\right\|_{L^{2}(\mathcal{G} \times \mathcal{Q})} \leq C_{1, \rho} e^{R L /} \rho^{-M} .
$$

Similarly,

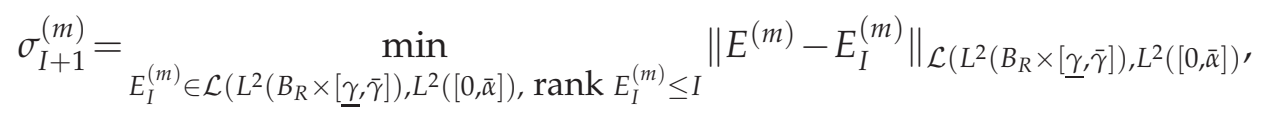


where

$$
\left(E^{(m)} \psi\right)(\alpha)=\int_{B_{R} \times[\underline{\gamma}, \vec{\gamma}]} \varphi^{(m)}(\vec{a}, \alpha, \gamma) \psi(\vec{a}, \gamma) d \vec{a} d \gamma, \quad \forall \alpha \in[0, \bar{\alpha}] .
$$

Then

$$
\sigma_{I+1}^{(m)} \leq\left\|E^{(m)}-\tilde{E}_{I}^{(m)}\right\|_{\mathcal{L}\left(L^{2}\left(B_{R} \times[\underline{\gamma}, \bar{\gamma}]\right), L^{2}([0, \bar{\alpha}])\right.}=\left\|\varphi^{(m)}-r_{I}^{(m)}\right\|_{L^{2}\left(B_{R} \times[\underline{\gamma}, \bar{\gamma}] \times[0, \bar{\alpha}]\right)},
$$

where

$$
\left(\tilde{E}_{I}^{(m)} \psi\right)(\alpha)=\int_{B_{R} \times[\underline{\gamma}, \bar{\gamma}]} r_{I, \vec{a}, \alpha}^{(m)}(\gamma, \alpha) \psi(\vec{a}, \gamma) d \vec{a} d \gamma, \quad \forall \alpha \in[0, \bar{\alpha}] .
$$

Let us assume that the $\varphi^{(m)}$ satisfy the additional boundedness property

$$
\sup _{(\vec{a}, \alpha) \in B_{R} \times[0, \bar{\alpha}], m=0,1, \cdots}\left\|\varphi_{m}(\vec{a}, \cdot, \alpha)\right\|_{L^{\infty}\left(\mathcal{E}_{C}\right)} \leq K_{\rho} e^{R L / \underline{\gamma}},
$$

for some constant $K_{\rho}$, possibly unbounded as $\rho \rightarrow 0$. Then, in view of estimate (3.7),

$$
\sigma_{I+1}^{(m)} \leq\left\|\varphi_{m}-r_{I}^{(m)}\right\|_{L^{2}\left(B_{R} \times[\underline{\gamma}, \bar{\gamma}] \times[0, \bar{\alpha}]\right)} \leq C_{2, \rho} e^{R L / \underline{\gamma}} \rho^{-I} .
$$

Similarly,

$$
\sigma_{J+1}^{(m, i)} \leq\left\|G^{(m, i)}-G_{J}^{(m, i)}\right\|_{\mathcal{L}\left(L^{2}\left(B_{R}\right), L^{2}(\underline{\gamma}, \bar{\gamma})\right)},
$$

where the operators $G^{(m, i)}$ and $G_{J}^{(m, i)}$ are defined by

$$
\left(G^{(m, i)} \kappa\right)(\gamma)=\int_{B_{R}} z_{i}^{(m)}(\vec{a}, \gamma) \kappa(\vec{a}) d \gamma, \quad\left(G_{J}^{(m, i)} \kappa\right)(\gamma)=\int_{B_{R}} s_{J, \vec{a}}^{(m, i)}(\gamma) \kappa(\vec{a}) d \gamma
$$

Assuming

it follows

$$
\sup _{\vec{a} \in B_{R}, i, m=0,1, \cdots}\left\|z_{i}^{(m)}(\vec{a}, \cdot)\right\|_{L^{\infty}\left(\mathcal{E}_{C}\right)} \leq K_{\rho} e^{R L / \underline{\gamma}}
$$

$$
\sigma_{J+1}^{(m, i)} \leq\left\|z_{i}^{(m)}-s_{J, \vec{a}}^{(m, i)}\right\|_{L^{2}\left(B_{R} \times[\underline{\gamma}, \bar{\gamma}]\right)} \leq C_{3, \rho} e^{R L / \underline{\gamma}} \rho^{-J} .
$$

If estimates (5.10), (5.13) and (5.14) are sharp, in the sense that they asymptotically are identities, it holds

$$
\tau_{j}^{(m, i)} \simeq A_{\rho} \rho^{-(m+i+j)}
$$

for some constant $A_{\rho}$, where the symbol $\simeq$ means that $\tau_{j}^{(m, i)} / \rho^{-(m+i+j)} \rightarrow A_{\rho}$ as $\min \{m, i, j\} \rightarrow \infty$. Then, property (5.3) holds. Therefore, as we have $n=4$ parameters, estimate (5.8) yields

$$
\left\|u-u_{\left(M_{1}, M_{2}, M_{3}\right)}\right\|_{L^{2}(\mathcal{G} \times \mathcal{Q})} \leq D e^{R L / \underline{\gamma}} \sqrt[3]{L_{K}} \rho^{-\sqrt[3]{L_{K}}}
$$

for some constant $D>0$, where we assume that $M+I+J=K$. Note that in this case $L_{K} \simeq \frac{1}{3} K^{3}$, so we conclude that

$$
\left\|u-u_{\left(M_{1}, M_{2}, M_{3}\right)}\right\|_{L^{2}(\mathcal{G} \times \mathcal{Q})} \leq D e^{R L /} \underline{\gamma}(M+I+J) \rho^{-\sqrt[3]{3}(M+I+J)} .
$$




\subsection{Practical implementation}

In this subsection we analyze the determination of the number of modes required to meet a prescribed accuracy by the truncated RPOD expansion. This is based upon the following lemma.

Lemma 5.1. Assume that the coefficients of the RPOD expansion (2.4) satisfy the estimates

$$
\sum_{i_{1}>I_{1}}\left|\sigma_{i_{1}}\right|^{2} \leq \Sigma_{1}, \quad \sum_{i_{k}>I_{k}^{\left(i_{1}, \cdots, i_{k-1}\right)}}\left|\sigma_{i_{k}}^{\left(i_{1}, \cdots, i_{k-1}\right)}\right|^{2} \leq \Sigma_{k}, \quad k=2, \cdots, n-1 .
$$

Then, the following error estimate in $L^{2}$ norm holds:

$$
\left\|f-f_{P}\right\|_{L^{2}\left(\mathbf{X}_{1}^{n}\right)}^{2} \leq \Sigma_{1}+C_{P}\|f\|_{L^{2}\left(\mathbf{X}_{1}^{n}\right)^{\prime}}^{2}
$$

where $C_{P}=\Sigma_{2}+\cdots+\Sigma_{n-2}+\Sigma_{n-1}$.

We omit the proof of this lemma for brevity. Estimate (5.19) allows to derive a strategy for the practical computation of the RPOD expansion (2.4), if estimates (5.18) for the singular values are asymptotically sharp. Indeed assume that we want to determine the number of modes for truncation, $M, I$ and $J$ for the solution of the advection-diffusionreaction equation, such that

$$
\left\|u-u_{\left(M_{1}, M_{2}, M_{3}\right)}\right\|_{L^{2}(\mathcal{G} \times Q)} \leq \varepsilon
$$

for some targeted error $\varepsilon>0$. By estimate (5.19), it holds

$$
\left\|u-u_{\left(M_{1}, M_{2}, M_{3}\right)}\right\|_{L^{2}(\mathcal{G} \times Q)} \leq \Sigma_{1}+\left(\Sigma_{2}+\Sigma_{3}\right)\|u\|_{L^{2}(\mathcal{G} \times Q)}^{2}
$$

and

$$
\Sigma_{1}=\sum_{m \geq 1}\left|\sigma_{m}\right|^{2}, \quad \Sigma_{2}=\sup _{m \geq 1} \sum_{i \geq I+1}\left|\sigma_{i}^{(m)}\right|^{2}, \quad \Sigma_{3}=\sup _{m, i \geq 1} \sum_{j \geq J+1}\left|\sigma_{j}^{(m, i)}\right|^{2} .
$$

If estimates (5.10), (5.13) and (5.14) are sharp, then good estimators for $\Sigma_{1}, \Sigma_{2}$ and $\Sigma_{3}$ are provided by

$$
\Sigma_{1} \simeq S_{1}=\left|\sigma_{M+1}\right|^{2}, \quad \Sigma_{2} \simeq S_{2}=\left|\sigma_{I+1}^{(M)}\right|^{2}, \quad \Sigma_{3} \simeq S_{3}=\left|\sigma_{J+1}^{\left(M_{1}, M_{2}\right)}\right|^{2} .
$$

This suggests to recursively determine $M, I$ and $J$ in such a way that

$$
S_{1}<\varepsilon^{2} / 3, \quad S_{2}<\varepsilon^{2} /\left(3\|u\|_{L^{2}(\mathcal{G} \times Q)}^{2}\right), \quad S_{3}<\varepsilon^{2} /\left(3\|u\|_{L^{2}(\mathcal{G} \times Q)}^{2}\right) .
$$

This is possible if the truncated primary, secondary and tertiary expansions are constructed term by term, for instance by the power iteration algorithm (see [10]). 


\section{Numerical experiments}

This section is devoted to determine the effective convergence rate of the RPOD approximation of some analytic functions, so as of the solutions to the advection-diffusionreaction equation (4.1) when parameterized by the diffusivity, advection velocity and reaction coefficients.

\subsection{Numerical validation of RPOD expansion with toy models}

To assess the efficiency of the RPOD to approximate multidimensional data let us consider tensors sampled from the following test functions:

$$
\begin{aligned}
f_{1}\left(x_{1}, x_{2}, x_{3}, x_{4}, x_{5}\right)= & \sin \left(x_{1} x_{4} x_{5}^{2}\right)+\sqrt{9+x_{3}+4 x_{2}}, \\
f_{2}\left(x_{1}, x_{2}, x_{3}, x_{4}, x_{5}\right)= & \frac{1}{1+x_{1}+x_{2}+x_{3}+x_{4}+x_{5}}, \\
f_{3}\left(x_{1}, x_{2}, x_{3}, x_{4}, x_{5}\right)= & x_{1}^{2}\left\{\sin \left[5 x_{2} \pi+3 \log \left(x_{1}^{3}+x_{2}^{2}+x_{4}^{2}+x_{3}+\pi^{2}\right)\right]-1\right\}^{2} \\
& +\left(x_{1}+x_{3}-1\right)\left(2 x_{2}-x_{3}\right)\left(4 x_{5}-x_{4}\right) \cos \left[3 0 \left(x_{1}\right.\right. \\
& \left.\left.+x_{3}+x_{4}+x_{5}\right)\right] \log \left(6+x_{1}^{2} x_{2}^{2}+x_{3}^{3}\right) \\
& -4 x_{1}^{2} x_{2} x_{5}^{3}\left(1-x_{3}\right)^{3 / 2} .
\end{aligned}
$$

The spatial domain is the unit hypercube, and the toy model tensors are constructed by discretization these functions in a structured equispaced $10 \times 10 \times 10 \times 10 \times 10$-mesh. Thus, we have three 5-order tensors whose size is $10^{5}$. Note that $f_{1}$ and $f_{2}$ are fairly simple, but $f_{3}$ is more complex since it is constructed through algebraic operations plus composition of various transcendental functions. Also note that because of that term proportional to $\left(1-x_{3}\right)^{3 / 2}$, the function is not analytic.

Fig. 1 shows the $L^{2}\left(\mathbf{X}_{1}\right)$ errors of the RPOD expansion (5.8) in semi logarithmic scale for the three functions $f_{1}, f_{2}$ and $f_{3}$. All three curves are consistent with the exponential convergence, modified by the factor $L^{\frac{n-2}{2(n-1)}}$, that yields a concave curve for $L$ large enough. The concavity of the error curves is larger for smaller convergence rate, a characteristic of the function that yields the error estimate,

$$
e(L)=D_{n}^{\prime} L^{\frac{n-2}{2(n-1)}} \rho^{-L^{\frac{1}{n-1}}} .
$$

In order to test (6.4), which should hold for enough large values of $L$, we fit the two parameters $D_{n}^{\prime}$ and $\rho$. Specifically, in the fitting process, we consider for functions $f_{1}, f_{2}$ and $f_{3}$ the modes for which $L^{1 / 4}$ (in this case $n=5$ ) is larger than 2,3 and 6.5, respectively. The graphical result of this fit, shown in figure 1, confirms our expectations. In fact, in all the considered cases, the experimental values approach the ones found out by fitting the function (6.4). The values obtained for $\rho$ respectively are $4.54 \times 10^{4}, 1.06 \times 10^{2}$ and $1.26 \times 10^{4}$. These values are quite large, so the exponential factor in (6.4) is largely 


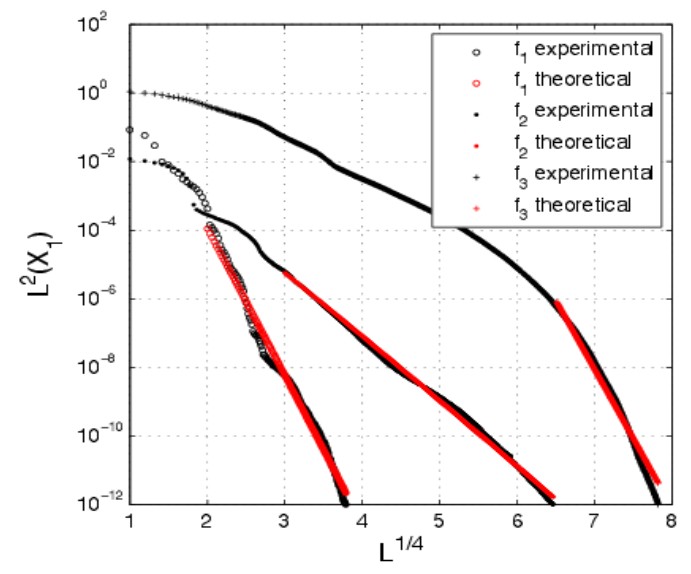

Figure 1: Convergence history of RPOD expansion error in logarithmic scale. In blue are plotted the experimental values and in red the theoretical ones. The x-axis corresponds to $L^{1 / 4}$, where $L$ is the number of modes $L$ in the re-arranged expansion.

dominant and the fitting curves are quite close to a straight line. These results are in good agreement with the error estimate (5.8), suggesting that it could be asymptotically sharp.

\subsection{Advection-diffusion-reaction equations}

In this sub-section we study the effective convergence rate of the RPOD approximation of the solution to de advection-diffusion-reaction equation. We assess the exponential convergence rate and investigate the variation of this rate with respect to the set $\mathcal{G}=$ $B_{R} \times[\underline{\gamma}, \bar{\gamma}] \times[\underline{\alpha}, \bar{\alpha}]$.

We consider the time-dependent advection-diffusion-reaction equation in the domain $\mathcal{Q}=(0,1) \times(0,1)$ and the source term and initial condition, given by

$$
f(t, x)=\sqrt{|x-t-0.3|}, \quad T_{0}(x)=|x-0.4| .
$$

These data have mild singularities, so the temperature solutions of (4.1), have a reduced regularity with respect to $x$ and $t$, in particular for $t=0$. The data we are considering to assess the efficiency of the RPOD approach are provided as a solution of the advectiondiffusion-reaction problem which is discretized by an implicit Euler second order scheme for the time derive. The advection term is approximated using an explicit second order Adams-Bashforth [15] scheme. Gauss-Lobatto-Legendre spectral method is used for the space approximation see [9]. Various integrals (with respect to either $\gamma, \alpha, \vec{a}$ or $x$ ) are computed using Gauss-Lobatto quadrature formulas with high resolution in the corresponding intervals. The numerical experiments are achieved using time step $\delta t=10^{-3}$ and the polynomial degree $N=24$. 
Test 1: Exponential convergence rate. Fig. 2 shows the convergence history of the RPOD expansion for the advection-diffusion-reaction equation in terms of the total number of modes in the expansion. We have considered the sets of diffusivities $\gamma \in[1,51]$, advection velocity $a \in[0,30]$ and reaction rates $\alpha \in[0,100]$. The error is measured in $L^{2}(\mathcal{G} \times Q)$ norm. The numbers of principal and secondary modes have been determined to be $\sigma_{m}^{\left(I_{m}+1\right)} \leq \varepsilon=10^{-5}$. The modes have been re-arranged in decreasing order of the effective singular values. We observe that the singular values indeed are good error estimators for this re-arranged expansion, as was argued in Subsection 5.2.
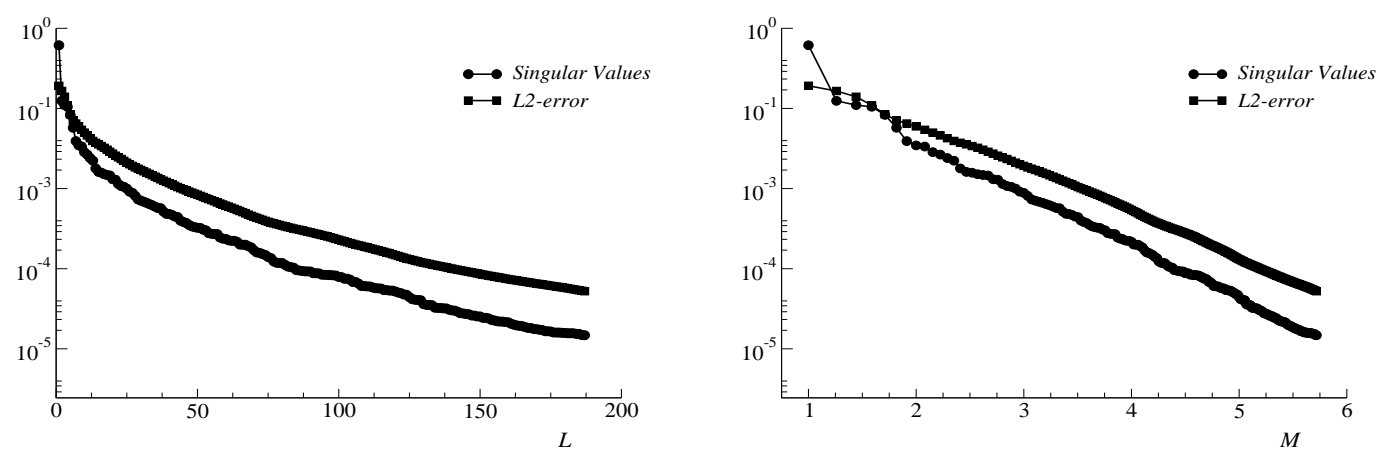

Figure 2: Convergence history for RPOD expansion of the solution of the advection-diffusion-reaction equation. Left : with respect to $L$, right with respect to $M=L^{1 / 3}$

Test 2: Dependence of the convergence rate with respect to the diffusion parameters range. The dependence with respect to the ratio of diffusivities $r=\bar{\gamma} / \gamma$ of the exponential convergence rate, stated by Theorem 3.1, is illustrated in Fig. 3. We depict the convergence history for our, computed for $r=2,10,25,100,400$ in all cases with a fixed interval of velocity advection $[\underline{a}, \bar{a}]=[0,5]$ and reaction rates $[\underline{\alpha}, \bar{\alpha}]=[0,10]$, with respect

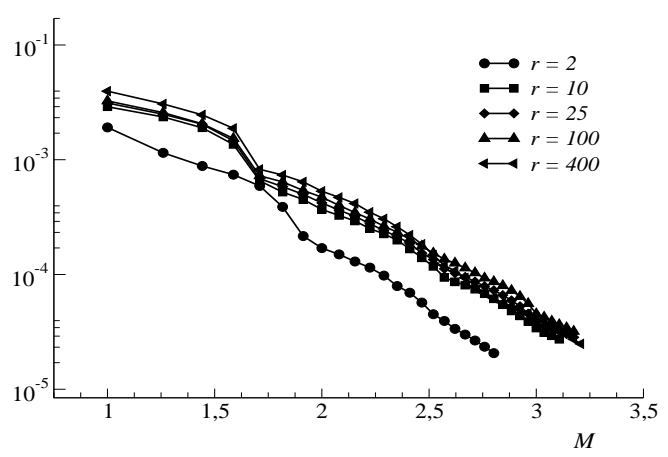

Figure 3: Variation of the RPOD errors (in logarithmic scale) with respect to the ratio $r=\bar{\gamma} / \underline{\gamma}$, for fixed $\left[a_{m}, \bar{a}\right]=[0,5]$ and $\underline{\alpha}=0, \bar{\alpha}=10$. The variable $M$ stands for $L^{1 / 3}$ where $L$ is the number of modes. 
to $M=L^{1 / 3}$. We can point out that the convergence rate degrades as $r$ increases. This is consistent with the dependence upon $r$ of estimate (4.7).

Test 3: Dependence of the convergence rate with respect to the reaction parameters range. We show in Fig. 4 the convergence rates history corresponding to $\underline{\alpha}=0, \bar{\alpha}=$ $1,25,50,75,100$ for fixed $\underline{\gamma}=1, \bar{\gamma}=51$ and $\underline{a}=0, \bar{a}=5$. We observe a decrease of the rate as $\bar{\alpha}$ increases, that however appears to be uniformly bounded, in agreement with estimate (4.7).

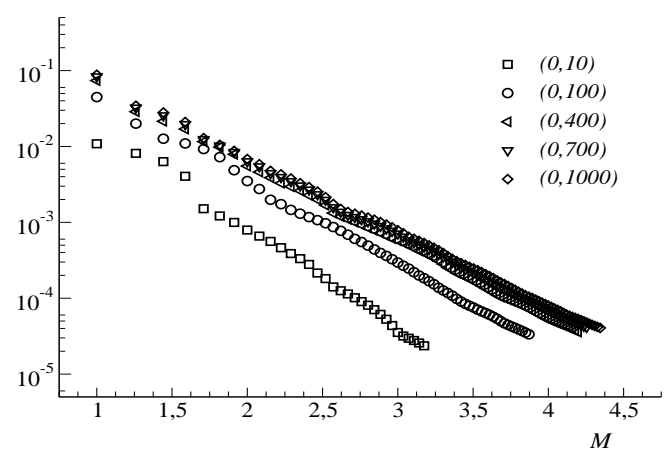

Figure 4: Variation of the RPOD errors with respect to the reaction rate $\alpha$. The curves correspond to $\underline{\alpha}=0$, $\bar{\alpha}=1,25,50,100,200$ with $\underline{\gamma}=1, \bar{\gamma}=51$ and $\underline{a}=0, \bar{a}=20$ in all cases. The variable $M$ stands for $L^{1 / 3}$ where $L$ is the number of modes.

Test 4: Dependence of the convergence rate with respect to the advection parameters range. We continue our study by testing the dependence of the convergence rate with respect to the range of the advection velocity $[\underline{a}, \bar{a}]$. We show in Fig. 5 the convergence rates history corresponding to $\underline{a}=0, \bar{a}=1,25,50,100,200$ for fixed $\underline{\gamma}=1, \bar{\gamma}=51$ and $\underline{\alpha}=0$,

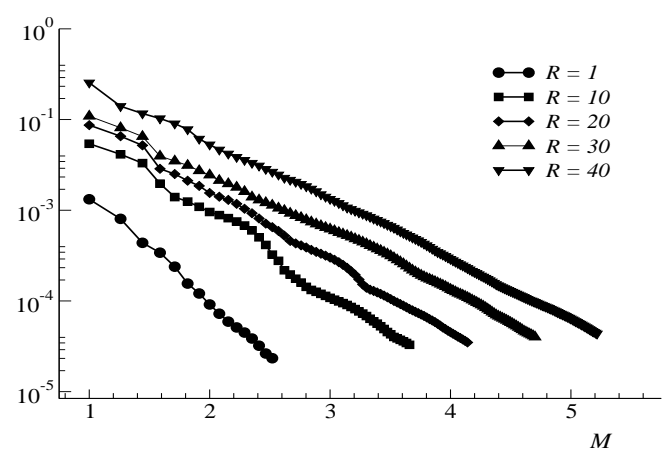

Figure 5: Variation of the RPOD errors with respect to the advection velocity variation $R=\bar{a}$. The curves correspond to $\underline{a}=0, R=1,10,20,30,40$ and 50 , with $\underline{\gamma}=50, \bar{\gamma}=101$ and $\underline{\alpha}=0, \bar{\alpha}=10$ in all cases. The variable $M$ stands for $L^{1 / 3}$ where $L$ is the number of modes. 
$\bar{\alpha}=50$. We can point out that the convergence rate degrades as $\bar{a}$ increases, but the slope of the convergence curves remains fixed. This also is strongly consistent with estimate (4.7).

The last numerical experiment studies whether the dependence of the exponential convergence on the diffusivities range $[\gamma, \bar{\gamma}]$ indeed takes place in terms of the ratio $r=\bar{\gamma} / \gamma$. This is confirmed by the result plotted in Fig. 6, where we consider the couples $(\underline{\gamma}, \bar{\gamma}) \equiv(1,2)$ and $(4,8)$, corresponding to $r=2$, and $(\underline{\gamma}, \bar{\gamma})=(1,25)$ and $(4,100)$, corresponding to $r=25$, with fixed $\underline{a}=0, \bar{a}=5$ and $\underline{\alpha}=0, \bar{\alpha}=10$. We observe that the slope of the convergence curve for $r=2$ is larger than for $r=25$ (for large values of $M$ ), in agreement with estimate (4.7).

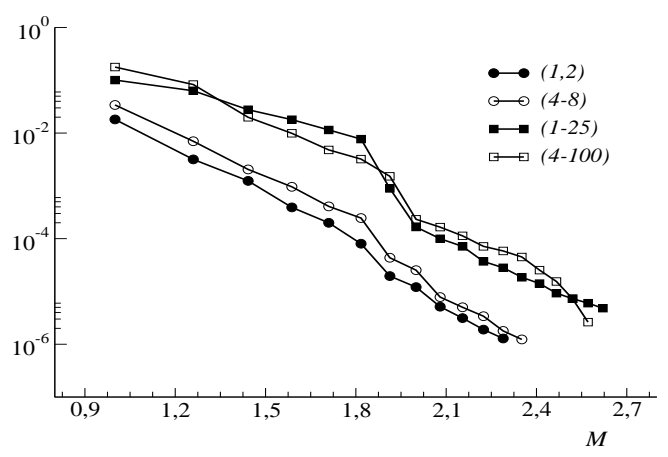

Figure 6: Analysis of dependence of the RPOD errors with respect to the ratio $r=\bar{\gamma} / \underline{\gamma}$. The curves correspond to the indicated pairs $(\underline{\gamma}, \bar{\gamma})$. The variable $M$ stands for $L^{1 / 3}$ where $L$ is the number of modes.

\section{Conclusion}

We have studied in this paper the RPOD expansion of the solutions of the advectionreaction-diffusion problem. We have demonstrated the exponential rate of convergence of the RPOD expansion for its solution. The proof is based upon the analyticity of its solution with respect to the parameters appearing in the equation.

We have also applied the RPOD to approximate analytic functions, that also show an exponential convergence rate.

We conclude the ability of the RPOD expansion to construct low-rank approximations of smooth multi-parametric functions, depending on a moderate number of parameters.

\section{Acknowledgments}

The research of Tomás Chacón has been partially supported by the Spanish Government - EU FEDER Fund project MTM2015-64577-C2-1-R, and by the IDEX program of the Université de Bordeaux. The research of Mejdi Azaiez has been partially supported by the 
Spanish Government EUFEDER Fund project MTM2015-64577-C2-1-R by NSFC/ANR joint program 51661135011. The research of Emma Perracchione is accomplished within Rete ITaliana di Approssimazione (RITA), GNCS-INdAM and supported by the research project Data Assimilation Methods for Machine Learning with application to environmental data, founded by UE ERA-PLANET GA n. 689443.

\section{References}

[1] R.A. Adams, J.J.F Fournier, Sobolev Spaces, Academic Press, 2003.

[2] D. V. Anosov, S. Kh. Aranson, V. I. Arnold, I. U. Bronshtein, V. Z. Grines, Yu. S. IL'YASHENKO, Ordinari Differential Equations and Smooth Dynamical Systems. Springer (1991).

[3] AZAï̈Z, M. AND BEN BeLGACEM, F., Karhunen-Loève's truncation error for bivariate functions, Computer Methods in Applied Mechanics and Engineering, Vol 290, pp 57-72, 2015

[4] M. Azaïez, F. Ben Belgacem and T. Chacón Rebollo., Error Bounds for POD expansions of parameterized transient temperatures CMAME. 305 (2016) 501-511

[5] M. AzaÏ̈z, F. Ben Belgacem, T. Chacón Rebollo, Recursive POD expansion for reactiondiffusion equation, Adv.Model. and Simul. in Eng. Sci. (2016) 3:3. DOI 10.1186/s40323-016-00601

[6] M. BAlajewicz, A new approach to model order reduction of the Navier-Stokes Equations, PhD thesis, Duke University, Durham, 2012.

[7] D. BELTON, Improving and extending the information on principal component analysis for local neighborhoods in 3D point clouds, The International Archives of the Photogrammetry, Remote Sensing and Spatial Information Sciences, 37 (2008), B5: $477 \mathrm{ff}$.

[8] G. BerKoOz, P. Holmes, J.L. Lumley, The proper orthogonal decomposition in the analysis of turbulent flows, Annu. Rev. Fluid Mech. 25 (1993), pp. 539-575.

[9] Bernardi, C. and Maday, Y., Approximations spectrales de problèmes aux limites elliptiques, Mathématiques et applications, Springer, Paris, Berlin, Heidelberg, 1992.

[10] F. Chinesta, A. Ammar, A. Leygue, R. Keunings, An overview of the proper generalized decomposition with applications in computational rheology, J. Non-Newtonian Fluid Mech. 166 (2011), pp. 578-592.

[11] A. CoHEn AND R. Devore, Kolmogorov widths under holomorphic mappings, IMAJ. Numer. Anal., 36 (2016), pp. 112.

[12] A. Cohen, R. Devore And CH. SCHWAB, Analytic regularity and polynomial approximation of parametric and stochastic elliptic PDEs, Analysis and Applications. Vol. 09, Issue 01, January 2011

[13] L. De Lathawer, B. De Moor, J. Vandewalle, On the best rank-1 and rank- $\left(R_{1}, R_{2}, \cdots, R_{N}\right)$ approximation of higher-order tensors, SIAM J. Matrix. Anal. Appl. 21 (2000), pp. 1324-1342.

[14] L. De LATHAWER, B. De MOOR, J. VANDEWALle, A multilinear singular value decomposition, SIAM J. Matrix. Anal. Appl. 21 (2000), pp. 1253-1278.

[15] C. A. J. Fletcher, Computational Techniques for Fluid Dynamics 1 Fundamental and General Techniques, Springer-Verlag. 1988.

[16] G.H. Golub, C.F. VAN LOAN, Matrix Computations, Johns Hopkins University Press, Baltimore, MD, 1996.

[17] C. Heyberger, P.A. BOUCARD, D. NÉRON, Multiparametric analysis within the proper generalized decomposition framework, Comput. Mech. 49 (2012), pp. 277-289.

[18] I.T. JolLIFFE, Principal Component Analysis, New York, Springer Verlag, 1986. 
[19] M. KiRBY, L. SiROVICH, Application of the Karhunen-Loève procedure for the characterization of human faces, IEEE Transactions on Pattern Analysis and Machine Intelligence, 12 (1990), pp. 103-108.

[20] G. LitTle, J. B. ReAdE, Eigenvalues of analytic kernels. SIAM J Math Anal. 15 (1984), pp. $133-6$.

[21] M.M. LoÈVE, Probability Theory, Van Nostrand, Princeton, NJ, 1988.

[22] J. MERCER, Functions of positive and negative type and their connection with the theory of integral equations, Phil. Trans. Royal Society, 209 (1909), pp. 415-446.

[23] M. MÜLler, On the POD Method: An abstract investigation with applications to reduced-order modeling and suboptimal control, PhD thesis, Georg-August Universität, Göttingen, 2008.

[24] Trefethen, L. N., Approximation theory and approximation practice. Software, Environments, and Tools., Society for Industrial and Applied Mathematics (SIAM), Philadel-phia, PA, 2013.

[25] S. ULLMANN, J. LANG, POD-Galerkin modeling and sparse-grid collocation for a natural convection problem with stochastic boundary conditions, Lecture Notes in Computational Science and Engineering 97, pp. 295-315.

[26] N. Vannieuwenhoven, R. VAdebril, K. Meerbergen A new truncation strategy for the higher-order singular value decomposition, SIAM J. SCi. Comput, Vol. 34, No. 2, pp. A1027A1052, 2012.

[27] S. VOLKWEIN, Proper Orthogonal Decomposition and Singular Value Decomposition. Rapport technique 153, Institut fr Mathematik, universit de Graz, 1999.

[28] H. WOLfGANG, Tensor spaces and numerical tensor calculus. Springer series in computational mathematics, V 42, 2012 